\title{
The Ureteral Lumen during Peristalsis
}

\author{
RUSSELL T. WOODBURNE AND JACK LAPIDES \\ Departments of Anatomy and Surgery, Section of Urology. \\ The University of Michigan Medical School, \\ Ann Arbor, Michigan
}

\begin{abstract}
This study concerns the size and shape of the ureteral lumen and an assessment of its enlargement during peristalsis. To stop the action of the living dog ureter, it was flooded with isopentane close to its freezing point $\left(-160^{\circ} \mathrm{C}\right)$. The ureter remained frozen until completion of all technical procedures. Photographs of the cut end of active ureters also provided evidence of its luminal characteristics and a movie was achieved of its continuous action. The totally collapsed lumen is stellate in form, epithelial surfaces being in virtual contact. As the lumen opens the points of the stars are last to go, but a square shape and finally a circle result. In a typical experiment, the collapsed lumen had a cross sectional area of $0.12 \mathrm{~mm}^{2}$, enlarging to $2 \mathrm{~mm}^{2}$ in diuresis. This $\times 17$ enlargement occurred over a $3 \mathrm{~cm}$ length of the peristaltic wave. Enlargement of the lumen is largely accommodated by thinning of the muscle coats and attenuation of the mucous membrane, there being relatively little gross enlargement of the total segment.

Direct photography of the cut end of the proximal segment of the ureter completely confirms the section studies.
\end{abstract}

As an aid in understanding abnormal conditions affecting the ureter, there is much current interest in its normal hydrodynamics. A little information has recently been forthcoming concerning peristalsis and discharge rate (Tsuchida, '69), but there appears to be no data in the literature bearing on the shape or size of the lumen in its varying functioning states. It is not difficult to observe the ureter during peristaltic activity in an anesthetized dog and its lumen can be studied and photographed after sectioning. But the production of objective data as to size and shape that represents the living, functioning characteristics of the tube presents difficulties.

Although there were a number of failures, success was achieved in many cases through the use of a quick freezing technique. With ureteral peristalsis going on, a section of the tube in action was rapidly flooded with isopentane brought close to its freezing point by immersion in liquid nitrogen. Isopentane (2-methylbutane) freezes at $-160^{\circ} \mathrm{C}$, and remains liquid till close to that temperature. After flooding and almost instantaneous freezing, the section of ureter was quickly removed and immersed in the same fluid. Transferred to a freezing storage area, it was subsequently cut into blocks and these were sectioned on a freezing microtome. The sections were then stained and cleared on the slide. Intermittent serial sections through the various blocks allowed microscopic observations of luminal characteristics throughout the length of the segment undergoing peristalsis (figs. 1a-4a).

Analysis of the irregular luminal shapes that resulted presented some difficulties. No formulae or planimetric methods were found to be feasible to determine area for the luminal cross section. This was finally achieved by comparison to standard circular cross sectional areas through the weight relations of paper cut-outs. The straight line relationship between weight and area of standard circular cut-outs was expressed as a line graph and the crosssectional area of irregular luminal shapes was then derived through the weight of the cut-out. Standard "Aquabee" layout bond paper was used and the histological sections were projected to 100 diameters.

Many visual observations of the ureter in action were made. Since the peristaltic 

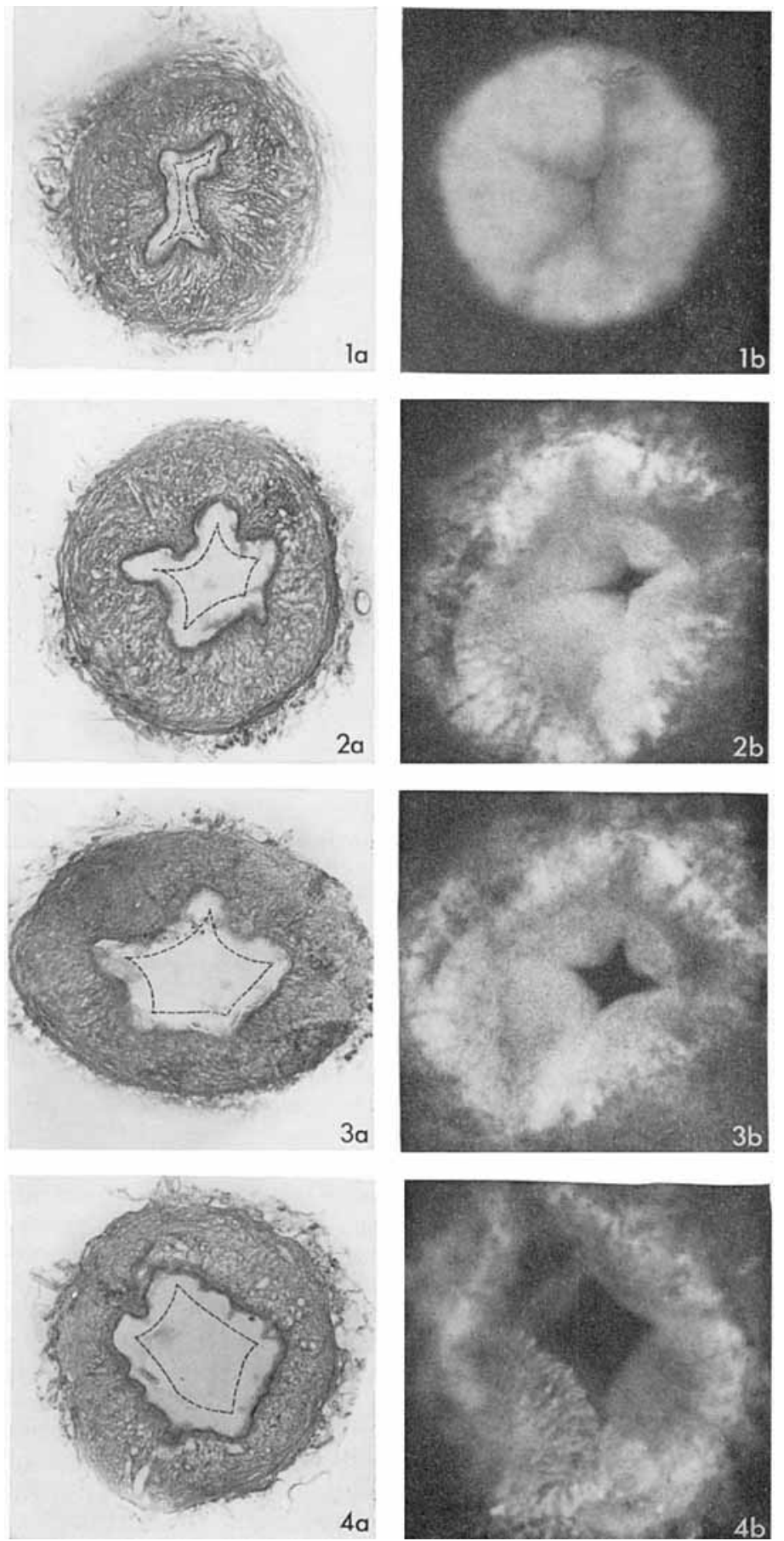

Figs. 1-4 Stages of ureteral opening.

(a) Photographs of sections at successive stages of opening. Since the mucous membrane was largely lost in the frozensection technique, dotted lines, based on remaining fragments of mucous membrane, reconstruct the lumen.

(b) Direct photographs of cut ends of the ureter in varying degrees of opening. 
wave moves through all segments of the ureter, its successive characteristics are visible at the cut end of a proximal segment. Peristalsis was usually stimulated with intravenous fluids and the cut end of the functioning ureter was photographed both in still and movie sequences (figs. $1 \mathrm{~b}-4 \mathrm{~b})$. A fragment of cover-slip placed over the end of the ureter materially reduced reflections for photography.

All observations agreed in depiction of the luminal shape at various stages. At the end of a peristaltic movement or at rest between movements the lumen is collapsed with its epithelial surfaces in contact. The lumen has a stellate form, five points being most commonly seen. Moderate urine flow altered this form only enough to provide freer passage of fluid, the shape usually losing one of its smaller sides to assume a four-pointed star. With greater flow the lumen opened to a square form with moderately curved sides. In full diuresis there was a progression to a circular lumen (figs. $1 \mathrm{~b}, 4 \mathrm{~b}, 6$ ).

Our data also provides information on the cross sectional area of the lumen under these differing degrees of enlargement. Although complete closure of the lumen doubtless occurs, the resting state in our material consistently showed a small starshaped or triangular lumen of about 0.12 $\mathrm{mm}^{2}$ area. Segments in full diuresis had a lumen of about $2 \mathrm{~mm}^{2}$ area. Thus, in this experiment, there was a 17 times enlargement from the resting phase of peristalsis to maximum diameter of the lumen. The chart (fig. 5) indicates for a length of $3 \mathrm{~cm}$, the peristaltic enlargement and wave form over the above range of cross sectional areas. The various luminal form relations are here also related to luminal size.

Our preparations indicate that the muscular wall thins during luminal enlargement and that luminal enlargement is only partially reflected in total diameter change of the enlarged segment. Mucous membrane attenuation also occurs during the enlargement. Photographs of open ends of a functioning ureter are presented (figs. 1b-4b) together with an artist's representation of the changes seen (fig. 6).

\section{DISCUSSION}

This study has revealed that the collapsed empty ureter of the dog has its epithelial surfaces in contact in a stellate configuration. In progressive opening of the tube the surfaces separate first to a square or diamond-shaped lumen and only with full dilation is a circular interior achieved.

It may be suggested that all relatively unsupported, thin-walled tubes are likely to follow similar patterns. Observation of

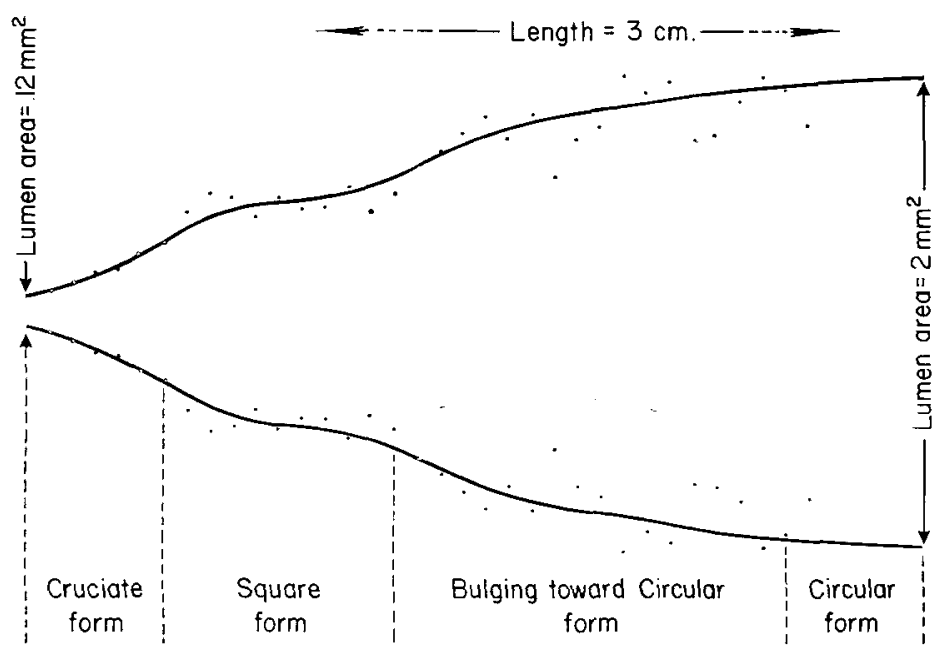

Fig. 5 Pictorial representation of one-half of a peristaltic wave, the lumen contracted to $0.12 \mathrm{~mm}^{2}$ cross sectional area and opened to a $2 \mathrm{~mm}^{2}$ cross sectional area. The luminal form is roughly related to luminal size. 


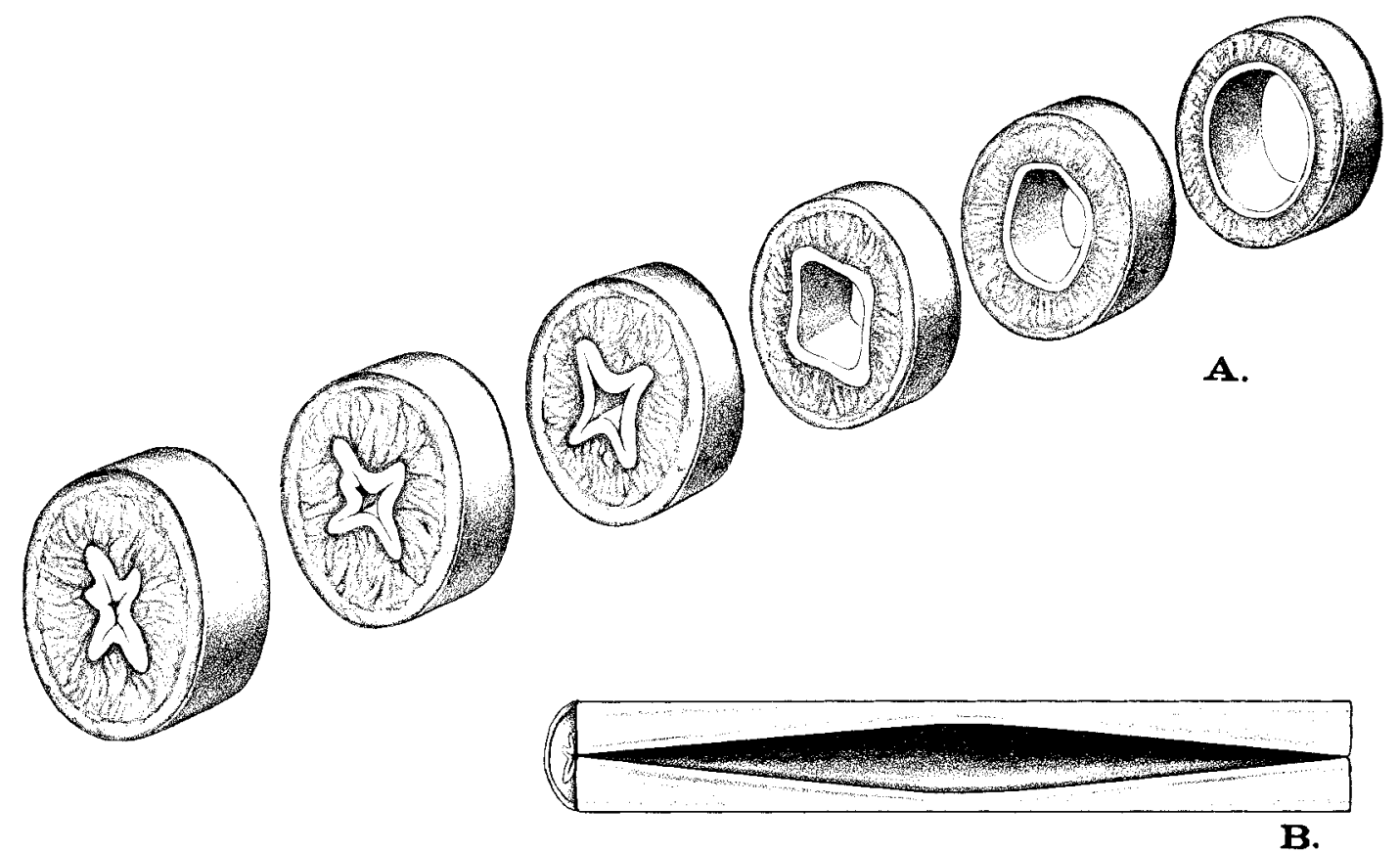

Fig. 6A Blocks of tissue represented successively to illustrate luminal change through a partial peristaltic wave.

Fig. $6 \mathrm{~B}$ Artist's representation of the full range of luminal change.

human frozen sections as used in various courses in cross-sectional anatomy indicate that there is a general rule here. The collapsed vagina has a rectangular shape with its anterior and posterior walls in contact. The rectum exhibits a highly infolded mucosa and, when empty, cross-sections of ascending and descending colon have a similar appearance. Even the more muscular esophagus exhibits marked infoldings when empty. The larger human ureter is remarkable for the filiform character of its mucosa when empty.

The study also indicates that the dog ureter has a great capacity for enlargement and suggests the participation of its structural parts in such enlargement.

\section{LITERATURE CITED}

Tsuchida, S. 1969 Computer analyses of urometrographic and electro-ureterographic data on the ureteral function at various urine flow rates. Tohoku. J. Exp. Med., 97: 297-310. 